\title{
Screening for duplications, deletions and a common intronic mutation detects $35 \%$ of second mutations in patients with USH2A monoallelic mutations on Sanger sequencing
}

Heather B Steele-Stallard ${ }^{1}$, Polona Le Quesne Stabej ${ }^{1}$, Eva Lenassi ${ }^{2,3}$, Linda M Luxon ${ }^{4,5}$, Mireille Claustres ${ }^{6,7}$, Anne-Francoise Roux ${ }^{6,7}$, Andrew R Webster ${ }^{2,3}$ and Maria Bitner-Glindzicz ${ }^{1,5,8^{*}}$

\begin{abstract}
Background: Usher Syndrome is the leading cause of inherited deaf-blindness. It is divided into three subtypes, of which the most common is Usher type 2, and the USH2A gene accounts for $75-80 \%$ of cases. Despite recent sequencing strategies, in our cohort a significant proportion of individuals with Usher type 2 have just one heterozygous disease-causing mutation in USH2A, or no convincing disease-causing mutations across nine Usher genes. The purpose of this study was to improve the molecular diagnosis in these families by screening USH2A for duplications, heterozygous deletions and a common pathogenic deep intronic variant USH2A: c.7595-2144A>G.
\end{abstract}

Methods: Forty-nine Usher type 2 or atypical Usher families who had missing mutations (mono-allelic USH2A or no mutations following Sanger sequencing of nine Usher genes) were screened for duplications/deletions using the USH2A SALSA MLPA reagent kit (MRC-Holland). Identification of USH2A: c.7595-2144A>G was achieved by Sanger sequencing. Mutations were confirmed by a combination of reverse transcription PCR using RNA extracted from nasal epithelial cells or fibroblasts, and by array comparative genomic hybridisation with sequencing across the genomic breakpoints.

Results: Eight mutations were identified in 23 Usher type 2 families (35\%) with one previously identified heterozygous disease-causing mutation in USH2A. These consisted of five heterozygous deletions, one duplication, and two heterozygous instances of the pathogenic variant USH2A: c.7595-2144A>G. No variants were found in the 15 Usher type 2 families with no previously identified disease-causing mutations. In 11 atypical families, none of whom had any previously identified convincing disease-causing mutations, the mutation USH2A: c.7595-2144A>G was identified in a heterozygous state in one family. All five deletions and the heterozygous duplication we report here are novel. This is the first time that a duplication in USH2A has been reported as a cause of Usher syndrome.

Conclusions: We found that 8 of 23 (35\%) of 'missing' mutations in Usher type 2 probands with only a single heterozygous USH2A mutation detected with Sanger sequencing could be attributed to deletions, duplications or a pathogenic deep intronic variant. Future mutation detection strategies and genetic counselling will need to take into account the prevalence of these types of mutations in order to provide a more comprehensive diagnostic service.

Keywords: Usher syndrome, USH2A, Deletion, Duplication, Pseudoexon, Multiplex ligation dependant probe amplification (MLPA), Array CGH

\footnotetext{
* Correspondence: maria.bitner@ucl.ac.uk

'UCL Institute of Child Health, London, UK

${ }^{5}$ UCL Ear Institute, London, UK

Full list of author information is available at the end of the article
} 


\section{Background}

Usher Syndrome is the leading cause of inherited deafblindness, accounting for $50 \%$ of cases. The disorder is clinically and genetically heterogeneous and is divided into three clinical subtypes, Usher type 1 (USH1), Usher type 2 (USH2) and Usher type 3 (USH3). Classification between subtypes is based upon type of hearing loss and presence or absence of vestibular dysfunction [1,2]. In type 1, affected people have profound congenital deafness, absent vestibular function and prepubertal onset of retinitis pigmentosa (RP); in type 2 the hearing loss is moderate to profound (sloping pattern) congenital hearing loss, with normal vestibular function and pre or post-pubertal onset RP; and in type 3 , hearing loss maybe pre- or post-lingual but progressive in course, with normal or abnormal vestibular function and often post-pubertal onset of RP. Individuals who do not have the usual phenotypes for each of these three subtypes are classed as atypical. Usher Syndrome is an autosomal recessive disorder. Previous studies have identified 12 loci and 10 causative genes. The most recently identified gene is CIB2, which is a rare cause of Usher Type 1 [3]. In addition to these 10 genes, PDZD7 has been implicated as both a modifier and a potential contributor to digenic inheritance [4].

The prevalence of Usher Syndrome has been estimated to be 3-6 per 100,000, although recently it has been reestimated to have a much higher frequency of 1 in 6,000 [5]. Usher type 2 is the most prevalent form accounting for more than half of reported cases. There are three genes underlying USH2: USH2A, GPR98 and DFNB31 (WHRN), with $U S H 2 A$ accounting for $75-80 \%$ of cases [6-8]. The mutational spectrum of $U S H 2 A$ is diverse and includes nonsense, frameshift, missense and spliceaffecting mutations, as well as deletions and small duplications [9]. Identifying the correct disease-causing variant is often confounded by the polymorphic nature of this gene, and the high frequency of novel mutations associated with this syndrome. For this reason, missense variants are assigned a value for their likelihood of causing disease ranging from Unclassified Variant 1 to 4 (UV1 to UV4). This classification system is based on frequency in controls, if the variant is novel in Usher syndrome, segregation with disease, and bioinformatic analysis of pathogenicity and conservation. UV4 variants are considered likely pathogenic, UV3 possibly pathogenic, UV2 possibly polymorphism, UV1 likely polymorphism $[7,10]$. Details of mutations and their ranked pathogenicity are recorded and revised in the Usher syndrome database, an invaluable tool in the molecular diagnosis of this disorder $[9,10]$.

Despite recent sequencing strategies that have analysed nine Usher Syndrome genes, and other studies involving thorough sequencing of $U S H 2 A, 8-19 \%$ of USH2 individuals have just one heterozygous likely diseasecausing mutation in USH $2 A$ [6-8], and $13 \%$ of USH2 patients have no convincing disease-causing mutations [7]. Unidentified mutations in USH2 individuals could lie in the promoter, regulatory regions, and deep intronic areas, all of which are not usually analysed during conventional mutation screening. Recently there has been a report of a pathogenic deep intronic variant USH2A: c.7595-2144A $>\mathrm{G}$ that causes the inclusion of a $152 \mathrm{bp}$ pseudoexon in the mRNA transcript, leading to the frameshift p.(Lys2532Thrfs*56) [11]. This was identified using investigation of RNA transcripts, underpinned by less invasive techniques for obtaining Usher gene RNA from affected individuals $[12,13]$.

Missing variants might also be attributable to heterozygous deletions and duplications, either those involving single or multiple exons, or the whole gene. Current investigations into the genetic basis of Usher syndrome have focused on Sanger sequencing to detect mutations $[7,8]$. It is not possible to robustly detect deletions and duplications by sequencing alone, as this method is not sensitive to relative changes in the copy number of exons. Homozygous whole single exon or multi-exon deletions can be inferred from consistent PCR nonamplification, but these are not very common, especially in the absence of consanguinity. Large duplications and heterozygous large exonic deletions are in effect 'invisible' if the breakpoints are outside of the amplified region. Previous work has identified such deletions through haplotype analysis, but to be comprehensive this requires the individual to carry informative variants in every exon. More recent methodologies have looked for deletions and duplications using array comparative genomic hybridization (array CGH) and multiplex ligation dependent probe amplification (MLPA) $[10,14,15]$. These methods have not yet been utilised to detect deletions and duplications in $\mathrm{USH} 2 \mathrm{~A}$ in USH2 and atypical individuals. As this gene is the major genetic contributor to USH2, and occasionally a cause of atypical Usher, it is important to screen for deletions and duplications in USH 2 A.

In this study we aimed to improve the molecular diagnosis of USH2 and atypical Usher by searching for deletions and duplications in USH2A by MLPA and array CGH, and by screening for the pathogenic deep intronic variant USH2A: c.7595-2144A>G. We also sought to develop methods to analyse splicing variants at the RNA level.

We identified 35\% of missing mutations in USH2 families with one previously identified (monoallelic) pathogenic/UV4/UV3 mutation in USH2A. These variants include five novel deletions in $U S H 2 A$ and one novel duplication. Results were confirmed by array $\mathrm{CGH}$, and where possible by RNA extracted from nasal epithelial cells and dermal fibroblasts. This is the first time that 
proband derived fibroblasts have been used for the study of splicing variants in Usher Syndrome.

\section{Methods}

\section{Patient and control DNAs}

Thirty-eight USH2 and 11 atypical families were included in this study. Twenty-three of these USH2 families had one pathogenic/UV4/UV3 mutation in USH2A. The remaining 15 USH2 families and 11 atypical families had no convincing disease-causing mutations (no mutations above UV2/UV1) in nine Usher genes, MYO7A, USH1C, CDH23, PCDH15, SANS, USH2A, DFNB31 (WHRN), GPR98, or CLRN1 (only CIB2 not analysed as CIB2 was described after the completion of this study). These probands were selected from 121 USH2 and 11 atypical families that were part of a previous mutation screening programme, the National Collaborative Usher Study [7]. For genotypes of the forty-nine families screened see Additional file 1. Clinical data for all atypical families, and USH2 families with mutations identified in this study, is given in Additional file 2. Informed consent was obtained from all participants. For nasal epithelial brushings and skin punch biopsies, additional informed consent for these procedures was given. Control DNA and RNA were obtained from consenting unrelated healthy individuals. This study adhered to the provisions of the declaration of Helsinki, and was approved by the National Research Ethics Committee London South East.

\section{Multiplex ligation dependent amplification (MLPA)}

The SALSA MLPA FAM labelled reagent kit with probe mixes P361-A1/ P362-A2 developed by MRC-Holland (MRC-Holland, Amsterdam, Netherlands), was used to detect deletions and duplications in the USH $2 \mathrm{~A}$ gene. Two MLPA probe mixes were required to encompass all 72 exons. Each probe mix contained 15 internal control probes; nine probes detected non-Usher genes on autosomes, four Q-oligonucleotides detected low DNA quantity and two D-oligonucleotides detected incomplete DNA denaturation. In addition to the $49 \mathrm{USH} 2$ and atypical families, parents in two further families were included to act as positive controls and confirm the validity of the method. These families were previously identified to have large homozygous deletions in $U S H 2 A$ [7]. Family 221 had a homozygous deletion of exon 47, and family 683 had a homozygous deletion of exon 5058 . The latter of these was previously published as a homozygous deletion of USH2A exons 50-55. MLPA analysis however showed this was in fact a deletion spanning exons 50-58, which was homozygous in the proband and heterozygous in both parents. One deletion control and one healthy control were run per 10 proband DNA samples. Controls without DNA, consisting of TE buffer were used to check for contamination in reagents.

Reactions were performed as per manufacturer's instructions [16]. Fragment size separation was conducted on the Applied Biosystems Inc. (ABI) 3730 DNA analyser with POP7 polymer capillary electrophoresis. Peak patterns were first evaluated using the raw data check list and the peak pattern evaluation flow chart available from MRC Holland (supplied as part of the MLPA general protocol). Data that passed peak pattern evaluation was normalised against negative controls, and dosage quotients for each probe calculated using Gene Marker v2.2.0 MLPA analysis software (SoftGenetics, Pennsylvania, USA) [17]. A probe dosage quotient value of less than 0.8 was considered a deletion, 0.8 to 1.2 normal, greater than 1.2 a duplication. Samples failed analysis if three or more of the nine supplied non-Usher gene control probes were deleted/duplicated.

\section{CGH array}

A custom designed CGH-microarray chip $(12 \times 135 \mathrm{k})$ that includes 16 sensorineural hearing loss genes was used on a high-resolution microarray platform according to the manufacturer's recommendations (Nimblegen; Roche Diagnostics, Basel, Switzerland). The CGH-microarray chip includes 77,366 probes covering the Usher-related genes (MYO7A, CDH23, PCDH15, USH1C, USH1G, USH2A, GPR98, DFNB31, PDZD7, USH3A) and their 10,000-bp 5' and 3' regions. The average probe length is 60 bases and the spacing between starts of probes covering exons and introns is $35 \mathrm{bp}$. The slides were scanned using InnoScan 900 A (Inopsys, Toulouse, France) and analyzed using Deva 1.2.1 software (Roche NimbleGen, Inc.).

\section{Deletion breakpoint mapping on genomic DNA}

Primers were designed to amplify across deletion breakpoints, based on array CGH results. PCR and sequencing was conducted with Biotaq DNA polymerase (Bioline, London, UK) and Big Dye terminator v1.1 (Applied Biosystems, Texas, USA). Primer sequences are available on request.

\section{Nasal epithelial brushings}

The lateral inferior turbinate was gently brushed five times with a $3 \mathrm{~mm}$ by $1.8 \mathrm{~mm}$ bronchial cytology brush (Diagmed Ltd, North Yorkshire, UK). Brushes were immediately placed in cell lysis buffer (supplied with NucleoSpin RNA II, Macherey-Nagel, Duren, Germany).

\section{Skin biopsies and fibroblast cell culture}

Punch biopsies were taken from consenting patients who carried USH2A: c.7595-2144A >G. Biopsied skin was placed in medium (DMEM glutamax + with $10 \%$ fetal bovine serum, 80 Units $/ \mathrm{ml}$ of Penicillin and $80 \mathrm{mg} / \mathrm{L}$ of 
Streptomycin). Fibroblasts were isolated and cultured according to standard procedures. The established fibroblast cell lines were trypsinised and passaged weekly as per standard protocols. For RNA extraction cells in the log phase of growth were pelleted and resuspended in cell lysis buffer (supplied with NucleoSpin RNA II, Macherey-Nagel, Duren, Germany).

\section{RNA extraction and CDNA synthesis}

RNA was extracted from fibroblast cell pellets and nasal epithelial cells with NucleoSpin RNA II (MachereyNagel, Duren, Germany) as per manufacturer's instructions. RNA was quantified and assessed for purity using Nanodrop 1000. For cDNA synthesis $2 \mu \mathrm{g}$ of RNA was used for fibroblast samples, and $0.8 \mu \mathrm{g}$ to $1 \mu \mathrm{g}$ was used for nasal samples. cDNA was synthesised using the Bioline cDNA synthesis kit.

\section{Reverse transcription PCR (RT-PCR)}

Genomic contamination was assessed in cDNA using intron-spanning primers for GAPDH. In addition to this all RT-PCR primers were designed to be intronspanning. When necessary PCR reactions were nested with internal primers. Primer sequences are available on request.

\section{Accession numbers}

Variants are described as per Human Genome Variation Society's recommendations, where +1 is the $\mathrm{A}$ in the ATG translation start codon of USH $2 A$ accession number NM_206933.2.

\section{Results}

Identification of large genomic rearrangements in the USH $2 A$ gene

Forty-nine families without convincing biallelic diseasecausing variants were screened for deletions and duplications in $U S H 2 A$ by MLPA. This group of families consisted of 23 USH2 families with one previously published pathogenic/UV4/UV3 mutation in USH2A, and 15 USH2 and 11 atypical families with only UV2/UV1, or no variants, across 9 Usher genes (i.e. no convincing disease-causing variants). For genotypes of all these families see Additional file 1.

No deletions or duplications were found in the 15 USH2 families or in the 11 atypical families with no convincing disease causing mutations. In the 23 families with a published monoallelic pathogenic/UV4/UV3 mutation in $U S H 2 A$, seven families were identified with heterozygous deletions or duplications. In order to exclude the possibility that genetic variants beneath the MLPA oligonucleotides might be causing lack of annealing of probes to target sequences, these regions were checked by Sanger sequencing in the seven identified families for variants. In one family a heterozygous mutation was found underneath the MLPA probe binding site of the 'deleted' target exon, and this was considered to be a false positive result. The remaining six cases consisted of five families with heterozygous deletions, and one family with a heterozygous duplication. The genotypes of these families are shown in Table 1. Segregation analysis in each of these families showed that all deletions or duplications were in trans with a previously identified pathogenic variant (Figure 1A). For deleted/duplicated MLPA probe values in these families, see Additional file 3.

The heterozygous duplication of exons 4 to 13 identified in family 283 is novel. To our knowledge this is the first time that a duplication of one or more exons in USH $2 A$ has been identified in Usher syndrome. This duplication is in trans with p.(Glu767Serfs*21) and segregates with disease in the family. All five identified deletions were also novel when checked against the Usher Syndrome mutation database [9]; there have been no previous reports of deletions of $U S H 2 A$ exon 4 , exon 27, exon 40 and exon 70. There is one previously reported deletion of $U S H 2 A$ exons 22-23, however recent investigations have revised the deletion in that family to USH $2 A$ exons 22-24 [6,9]. The deletion of USH $2 A$ exons 22-23 identified in our study is therefore novel.

\section{Confirmation of USH2A large genomic rearrangements and breakpoint mapping}

To further confirm and study the effects of the identified deletions, we isolated RNA from nasal epithelial cells from probands in two families. The proband from family 151 carried a deletion of $U S H 2 A$ exon $22-23$ and p. (Gln1063Serfs*15) in a compound heterozygous state, and the proband from family 657 carried a deletion of exon 70 and p.(Arg63\%) in a compound heterozygous state.

Reverse transcription PCR (RT-PCR) amplification between $U S H 2 A$ exons $21-26$ in the cDNA of the proband from family 151 produced bands of varying intensity, shown in Figure 2A. One band of 772 bp was observed in control cDNA, and corresponded to the non-deletion allele. A smaller band of 514bp was observed in the proband from family 151 and not on control cDNA; this was the expected size for a deletion of USH $2 A$ exons 22-23. Multiple attempts at sequencing this band failed. The deletion of USH2A ex22-23 is expected to be in frame and does not appear to be subject to nonsense mediated decay. The non-deleted allele in this individual amplified very faintly, and it is suspected that this allele, USH2A: p.(Gln1063Serfs*15), could be subject to nonsense mediated decay or there could be preferential amplification of the short PCR product from the deletion allele.

In the proband from family 657, repeated RT-PCR attempts to amplify $U S H 2 A$ cDNA across exon 68 to 71 
Table 1 Genotypes of probands identified with USH2A deletions/duplications and c.7595-2144A>G p.(Lys2532Thrfs*56)

\begin{tabular}{|c|c|c|c|c|c|c|c|c|c|}
\hline Family & Diagnosis & Gene & Allele $1^{\text {a }}$ & $\begin{array}{l}\text { Allele } 1 \text { predicted } \\
\text { protein change }^{\mathrm{a}}\end{array}$ & Allele $2^{b}$ & $\begin{array}{l}\text { Allele } 2 \text { predicted } \\
\text { protein change }^{b}\end{array}$ & $\begin{array}{l}\text { Pathogenicity } \\
\text { allele } 1^{\mathrm{a}}\end{array}$ & $\begin{array}{l}\text { Pathogenicity } \\
\text { allele } 2^{\mathbf{b}}\end{array}$ & Ethnicity ${ }^{c}$ \\
\hline 46 & USH2 & USH2A & c.6862G>T & p.(Glu2288*) & exon 40 deleted c.7452-68_7594+942del & p.(Leu2485Thrfs*25) & Pathogenic & Pathogenic & Caucasian \\
\hline 148 & USH2 & USH2A & c.2299delG & p.(Glu767Serfs*21) & $\begin{array}{c}\text { exon } 27 \text { deleted c.[5299-932_5572+1023del; } \\
\left.5572+1100 \_5573-1099 \mathrm{del}\right]\end{array}$ & p.(Met1767Valfs*6) & Pathogenic & Pathogenic & Caucasian \\
\hline 151 & USH2 & USH2A & c.3187_3188delCA & p.(Gln1063Serfs*15) & $\begin{array}{c}\text { exon 22-23 deleted c.4628-15914_4885 } \\
+472 \text { del }\end{array}$ & $\begin{array}{c}\text { p. } \\
\text { (Ile1544_Gly1629del) }\end{array}$ & Pathogenic & Pathogenic & Caucasian \\
\hline 309 & USH2 & USH2A & c.2299delG & p.(Glu767Serfs*21) & exon 4 deleted c.781_784+1375del & p.? & Pathogenic & Pathogenic & Caucasian \\
\hline 657 & USH2 & USH2A & c.187C>T & p.(Arg63*) & exon 70 deleted c.15053-26_15298-708del & p.(Leu5019Valfs*77) & Pathogenic & Pathogenic & Caucasian \\
\hline 283 & USH2 & USH2A & c.2299delG & p.(Glu767Serfs*21) & exons $4-13$ duplicated & p.? & Pathogenic & Pathogenic & Caucasian \\
\hline 24 & USH2 & USH2A & c.2299delG & p.(Glu767Serfs*21) & c.7595-2144A>G & p.(Lys2532Thrfs*56) & Pathogenic & Pathogenic & Caucasian \\
\hline 707 & USH2 & USH2A & c.2299delG & p.(Glu767Serfs*21) & c.7595-2144A>G & p.(Lys2532Thrfs*56) & Pathogenic & Pathogenic & Caucasian \\
\hline 128 & $\begin{array}{c}\text { Atypical } \\
\text { Usher }\end{array}$ & USH2A & Unknown & Unknown & c.7595-2144A>G & p.(Lys2532Thrfs*56) & Pathogenic & & \\
\hline
\end{tabular}

Variants in bold are novel mutations, identified in this study.

${ }^{a}$ All allele 1 variants have been previously published in these individuals [7].

${ }^{\mathrm{b}}$ All allele 2 variants were identified in this study by USH2A MLPA or sequencing for USH2A: c.7595-2144A>G.

'Caucasian represents UK and European. 


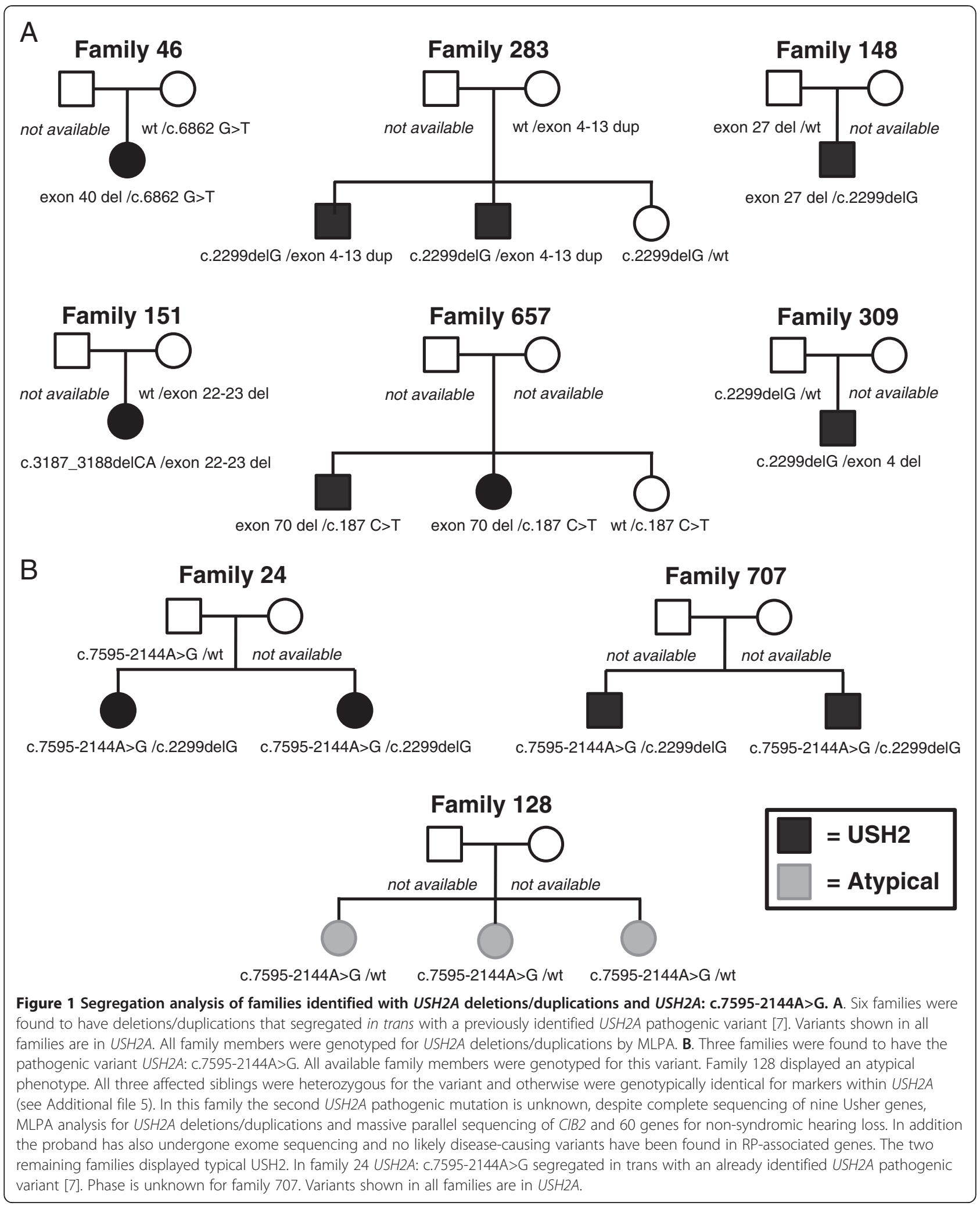

failed, but amplified in control nasal cDNA samples run during the same reaction. Additional RT-PCR reactions across $U S H 2 A$ exons $21-26$ also amplified in controls, but not in the proband from family 657 for either $U S H 2 A$ allele. The amount of RNA used for cDNA synthesis was more than for other probands who did amplify. To assess 

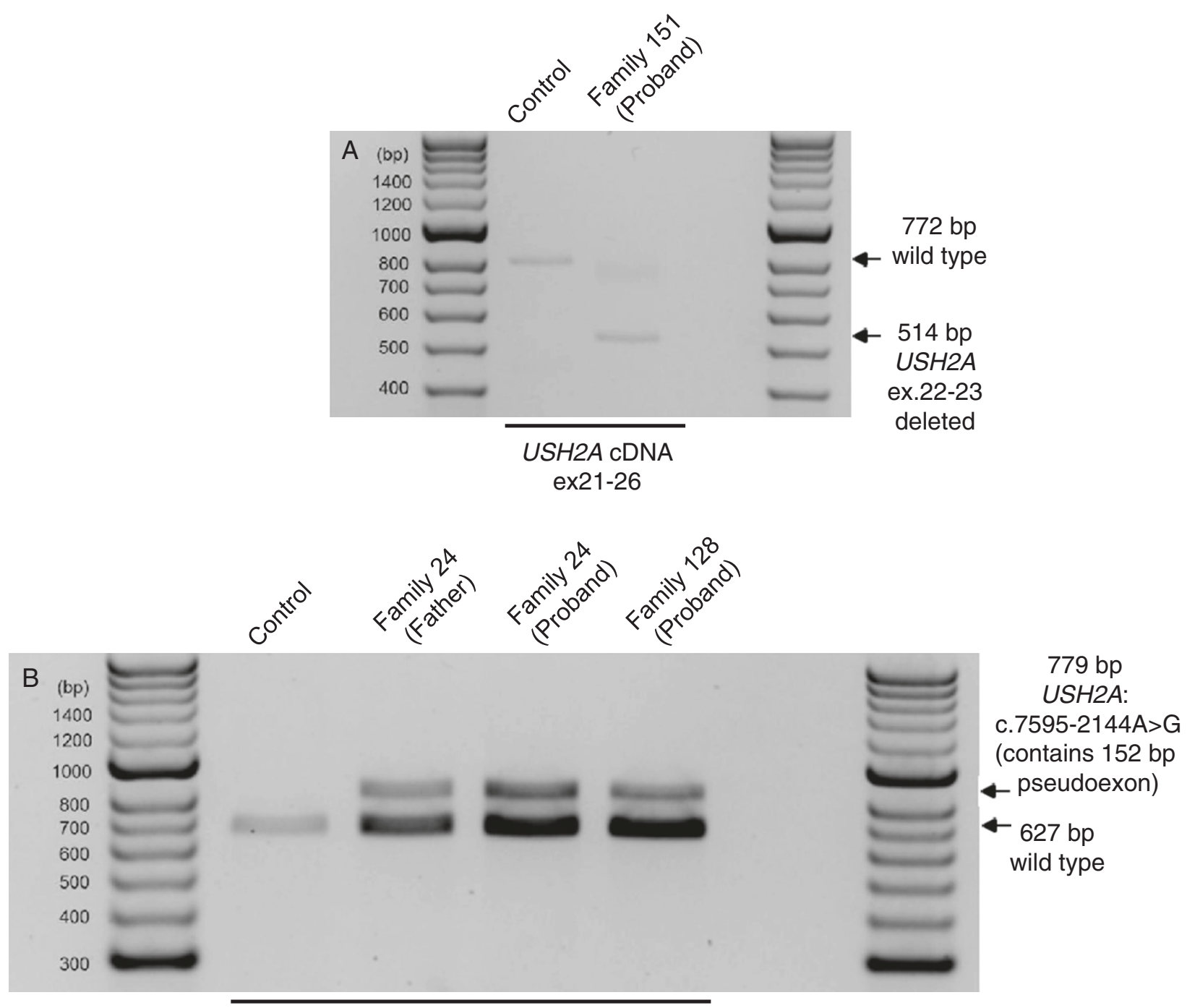

USH2A cDNA ex40-41

Figure 2 Reverse transcription PCR confirmation of USH2A exon deletions and USH2A: c.7595-2144A>G. A. Reverse Transcription PCR (RT-PCR) of USH2A exons 21-26 using RNA extracted from nasal epithelial cells as template. The proband from family 151 carries a deletion of USH2A exons 22-23 in trans with USH2A: p.(GIn1063Serfs*15). In this individual RT-PCR produced a shorter product of 514bp corresponding to a 258bp deletion of USH2A exons 22-23. The other allele in the person amplifies faintly. Amplification on control template produced a band of $772 \mathrm{bp}$, corresponding to wild type. B. Reverse Transcription PCR (RT-PCR) of USH2A exons 40-41 using RNA extracted from fibroblasts as template. The proband and father in family 24 , and the proband in family 128 all carry USH2A: c.7595-2144A>G. This variant is in USH2A intron 40, and has been previously reported to lead to the inclusion of a 152bp pseudoexon between exon 40-41 resulting in the pathogenic frameshift p.(Lys2532Thrfs*56). The proband in family 24 also carries this variant in trans with USH2A: p.(Glu767Serfs*21). The other disease-causing allele in family 128 could not be identified.

if PCR failure was limited to $U S H 2 A$, RT-PCR reactions for three other genes were conducted in this individual using the same template RNA sample. Amplification of two Usher genes GPR98, WHRN, and of the house-keeping gene GAPDH produced RT-PCR products of expected size in the proband from family 657 . This suggests that both alleles in this individual, exon 70 deleted (which is predicted to produce the frameshift p.(Leu5019Valfs*77)) and p. (Arg63*) produce $U S H 2 A$ long isoform transcripts that are subject to nonsense mediated decay.
A custom microarray was used to guide deletion breakpoint mapping and to confirm presence of deletions where no cDNA was available. Based on the probe coordinates, it is likely that the four single exon deletions, del E4, E27, E40 and E70 range approximately from 1.2 to $5 \mathrm{~kb}$ in length. The double exon deletion del E22-23 was found to be approximately $25 \mathrm{~kb}$, and the duplication dup E4-13 approximately $144 \mathrm{~kb}$ in length. The microarray coordinates were used to design PCR primers to amplify across the genomic breakpoints of 
the five identified deletions. The observed PCR products in all individuals carrying deletions were smaller than expected for wild type alleles. Subsequent sequencing of the PCR products successfully identified the breakpoints for all five deletions, shown in Table 1 . Interestingly the deletion of $U S H 2 A$ exon 27 in family 148 was found to comprise two deletions in close proximity (76bp apart) carried on the same allele USH2A: c.[5299-932_5572+ 1023del; 5572+1100_5573-1099del]. The deletion of exon 4 in family 309 , was found to be a partial exon deletion comprising the last $4 \mathrm{bp}$ of exon 4 and extending into intron 4, USH2A: c.781_784+1375del. Deletion mapping in this family is shown in Figure 3. For all other families see Additional file 4.

Sequencing for USH2A: c.7595-2144A $>$ G p.(Lys2532Thrfs*56) Of the 23 USH2 families with one pathogenic/UV4/UV3 mutation in $U S H 2 A$, we identified two USH2 families with the previously reported pathogenic variant $U S H 2 A$ : c.7595-2144A>G [11]. The mutation was in trans with
USH2A: p.(Glu767Serfs*21) in family 24 . In family 707 phase is unknown, but an affected sibling carried the same two pathogenic mutations (Figure 1B). USH2A: c.7595-2144A $>$ G was not found in the 15 USH2 families who had only UV2/UV1 variants.

Interestingly, one atypical family was also identified to carry USH2A: c.7595-2144A>G. This family consisted of three affected siblings, all with adult onset hearing loss (in the fourth decade), diagnosed after the onset of RP in the mid-teenage years. Vestibular function was normal in one sibling who has been tested. All three sisters were heterozygous for the mutation. The other diseasecausing variant in this family is unknown, with no other pathogenic/UV4/UV3 mutations identified in any Usher syndrome gene. Recent analysis of CIB2 and 60 genes causing non-syndromic hearing loss by massive parallel sequencing did not identify any other pathogenic mutations in this family. The proband has also undergone exome sequencing and no likely disease-causing variants have been found in RP-associated genes - data not

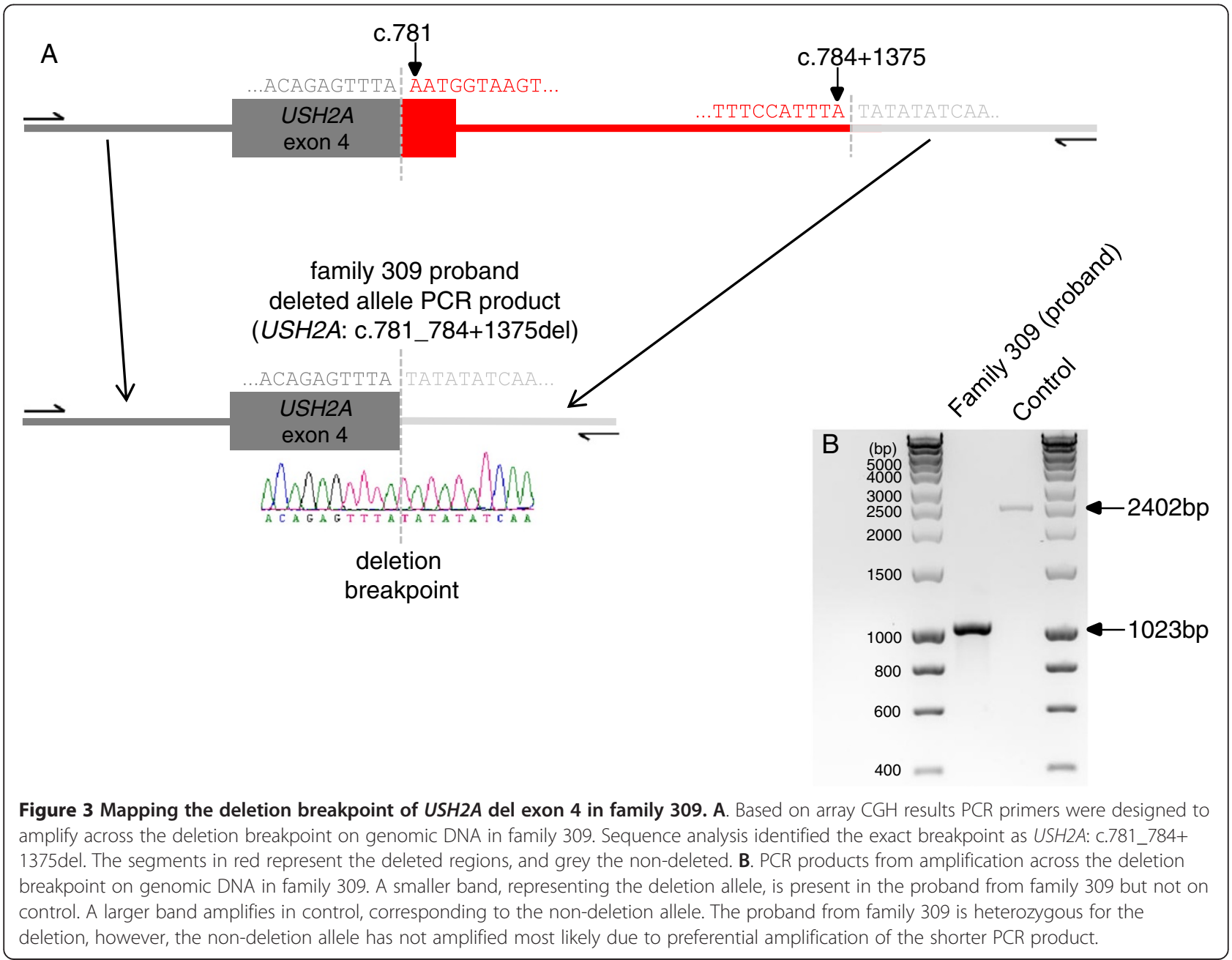


shown. Genotyping of polymorphic variants in these siblings showed they share USH2A haplotypes, shown in Additional file 5 .

\section{Confirmation of USH2A: c.7595-2144A>G on CDNA}

To further study and confirm reported effects of $U S H 2 A$ : c.7595-2144A $>$ G, we isolated dermal fibroblasts from family 24 and the atypical family 128 . RT-PCR to amplify USH $2 A$ exons 40-41, was conducted on cDNA synthesised from these cells. All heterozygous individuals produced a band of the expected size, $627 \mathrm{bp}$, plus an additional band of 779 bp that was not observed in controls, shown in Figure 2B. Gel extraction and sequencing of the bands showed the $627 \mathrm{bp}$ fragment corresponded to wild-type sequence. The sequence of the $779 \mathrm{bp}$ fragment contained a 152 bp sequence from intron 40, inserted between exon $40-41$. This sequence matches that previously reported by Vaché et al. [11] to be inserted at this point due to USH2A: c.7595-2144A>G. Our results confirm the findings of this previous paper regarding the splicing effects and likely pathogenicity of this deep intronic mutation.

\section{Discussion}

It is often not possible to identify both pathogenic variants in USH2A [6-8]. In this study we identified eight mutations in 23 USH2 families (35\%) who were known to have a monoallelic pathogenic/UV4/UV3 mutations in USH2A detected by Sanger sequencing. These consisted of five novel heterozygous deletions, one novel duplication in $U S H 2 A$, and two cases with the pathogenic variant USH2A: c.7595-2144A>G p.(Lys2532Thrfs"56). This is the first time that a duplication in $U S H 2 A$ has been reported as a cause of Usher syndrome.

Recently the observation of monoallelic mutations in an Usher gene has been attributed to digenic inheritance, with the second mutation residing in another Usher gene [18]. Two families reported by Bonnet et al. [18] were identified with one pathogenic allele in $U S H 2 A$, and one missense variant in an USH1 gene. As these changes in non- $U S H 2 A$ genes were missense variants and not definitely pathogenic alleles (nonsense, frameshifts or splice variants) they could in fact be rare benign variants, or disease modifiers. The true pathogenic mutation could be a second mutation within the $U S H 2 A$ gene which is not detected by a simple exon sequencing approach, such as those we describe here. In our 23 families with a monoallelic USH2A pathogenic mutation, $35 \%$ of second mutations were missed by the exon sequencing approach. Indeed, as Abu-Safieh et al. [19] argue in the case of Bardet-Biedl syndrome, oligogenic inheritance has not been conclusively substantiated for many diseases. Many cases of oligogenic inheritance are claimed when multiple genes are sequenced, and ethnic-specific, usually missense variants in a second gene are misinterpreted as pathogenic, as we have found in our previous study [7].

In $15 \mathrm{USH} 2$ families with no pathogenic/UV4/UV3 mutations we did not find any new variants. In five of these families UV2 variants had already been identified, but treated as not likely to be disease-causing (see Additional file 1). It is possible that initial mutation classification of these variants was too strict, and these variants are more likely to be disease-causing than their initial assessment. However due to the polymorphic nature of the Usher genes, and the lack of a convincing pathogenic second mutation it is not certain if these are rare polymorphic variants or pathogenic. They have therefore been given the cautious pathogenicity ranking of UV2.

Additional explanations for missing variants in families with zero or one convincing disease-causing mutation include mutations in introns or promoters that have pathogenic effects, or mutations in an as yet unidentified rare Usher gene. Nine Usher genes were analysed in these individuals, the $10^{\text {th }}$ Usher gene CIB2 was not sequenced; however this gene was documented to be a rare cause of USH1, and not the USH2 phenotype these families display. These individuals will require further investigation by exome sequencing to look for new genes, and/or sequencing of the entire genomic region or RNA analysis of known Usher genes in order to achieve a molecular diagnosis. Alternatively some may not have Usher syndrome per se, but could have disease caused by mutations in genes which cause non-syndromic deafness and non-syndromic RP, both of which are very genetically heterogeneous.

In 11 atypical families screened, we identified one pathogenic mutation $U S H 2 A$ : c.7595-2144A $>$ G in family 128. Previous studies have noted pathogenic mutations in Usher genes, in patients with atypical clinical features [8,20-22]. However in the absence of a second mutation we cannot be sure that $U S H 2 A$ is definitely the cause of the atypical Usher syndrome in this family. Nevertheless, despite thorough sequencing of nine Usher genes [7], $U S H 2 A$ MLPA, and additional screening in the proband for $C I B 2$, 60 genes causing non-syndromic hearing loss by massive parallel sequencing, and exome sequencing we were not able to identify any other obviously pathogenic mutations in this family. Further investigation of this family at the RNA level will be conducted in the future to help identify the missing variant.

In this study we have established that MLPA and RNA analysis can detect a significant proportion of mutations missed by exon sequencing. The nasal ciliated epithelial collection technique we use requires no anaesthetic, uses gentle brushing, lasts for a few seconds and has been very well tolerated by all participants. In addition we show for the first time that proband-derived fibroblasts can be used to study splice-affecting mutations in the 
clinically relevant $U S H 2 A$ long transcript. This technique has several useful implications. It is possible to transform fibroblasts into induced pluripotent stem cells, from which photoreceptors and retinal pigment epithelial cells can be derived [23]. This has recently been demonstrated with fibroblasts obtained from individuals with Usher syndrome and retinitis pigmentosa $[24,25]$. These cells could provide a basis for studying the biological effects of specific mutations and for testing the effects of potential new treatments currently being developed for Usher syndrome, such as small molecules or gene therapeutic approaches, in appropriate cells/cell-types [26-28]. This may help produce more translational relevant results as cells derived from patients with Usher Syndrome will have an advantage over current mouse models of USH1, which do not accurately represent the ophthalmic aspects of this disorder $[29,30]$.

In our previous Sanger sequencing effort of nine Usher syndrome genes in 121 USH2 families, we identified two pathogenic/UV4/UV3 variants in 65\% (79/121) of families, one pathogenic/UV4/UV3 variant in $21 \%$ $(26 / 121)$ of families and no pathogenic/UV4/UV3 variants in 13\% (16/121) of families. The USH2A MLPA kit we have used in this study was able to quantitatively detect changes in the copy number of an exon in a further 6 families and is therefore a useful addition in the molecular diagnosis of USH2. Taking into account all the new mutations identified and further work, these figures can be re-calculated for USH2 families. When sequenced for 9 Usher Syndrome genes, and screened for $U S H 2 A$ deletions, duplications and for the pathogenic variant $U S H 2 A$ : c.7595-2144A>G, 77\% (93/121) of USH2 families have two pathogenic/UV4/UV3 mutations, 11\% (13/121) have one pathogenic/UV4/UV3 mutation and $12 \%(15 / 121)$ have no pathogenic/UV4/UV3 variants. Considering just the $U S H 2 A$ gene, 11 families remain with mono-allelic $U S H 2 A$ mutations and a yet unidentified second mutation. A further search for intronic mutations, either by RNA analysis looking for abnormal splice variants or 'allelic drop-out', or high throughput sequencing of the USH $2 \mathrm{~A}$ genomic region, may be useful.

\section{Conclusions}

We found that 8 of 23 (35\%) mutations in individuals with a monoallelic mutation in $U S H 2 A$ can be attributed to deletions, duplications and a pathogenic deep intronic variant. Five novel deletions, one novel duplication and a previously-described intronic pathogenic mutation have been identified in this study using MLPA, sequencing and RNA analysis. Future mutation detection strategies and genetic counselling will need to take into account the prevalence of these types of mutations in order to provide a more comprehensive diagnostic service.

\section{Additional files}

\begin{abstract}
Additional file 1: Genotypes of all 49 families screened for USH2A deletions/duplications and USH2A: c.7595-2144A>G.

Additional file 2: Clinical data from all atypical individuals and USH2 probands with USH2A deletions, duplications and c.75952144A>G.

Additional file 3: MLPA probe values in families identified with USH2A deletion/duplications.

Additional file 4: Mapping of USH2A deletion breakpoints on genomic DNA.

Additional file 5: USH2A haplotype analysis of family 128.

\section{Abbreviations}

Array CGH: array comparative genomic hybridisation; MLPA: Multiplex ligation dependant probe amplification; RP: retinitis pigmentosa; RT-PCR: reverse transcription polymerase chain reaction; USH1: Usher Type 1; USH2: Usher Type 2; USH3: Usher Type 3; UV1: Unclassified variant 1 (likely polymorphism); UV2: Unclassified variant 2 (possibly polymorphism); UV3: Unclassified variant 3 (possibly disease-causing); UV4: Unclassified variant 4 (likely disease-causing).
\end{abstract}

\section{Competing interests}

The authors declare that they have no competing interests.

\section{Authors' contributions}

HS-S completed the laboratory work, and co-wrote the manuscript with MBG; MB-G supervised the laboratory work; EL and ARW reviewed all laboratory results and patient data and $L L$ reviewed all audiological data; $M C$ supervised the design of the array $C G H$ and AFR reviewed the CGH data. $L L$ and PLQS critically appraised the manuscript. All authors read and approved the final manuscript.

\section{Acknowledgements}

We thank Sarah Fielding and Brendan Martin in the North East Thames Regional Genetics Laboratory who provided technical help with MLPA, Lise Larrieu for technical help with the custom designed CGH-microarray chip, and Dr Amelia Shoemark for help with the nasal epithelial brushing technique. We would like to also thank the Combined Biomedical Research Centres of UCLH, Great Ormond Street Hospital and Moorfields Eye Hospital Sense, Deafness Research UK and Action on Hearing Loss. MB-G is supported by Great Ormond Street Hospital Children's Charity (GOSHCC). None of the funding bodies had any role in study design or data interpretation or in the writing or submission of the manuscript.

\section{Author details}

${ }^{1}$ UCL Institute of Child Health, London, UK. ${ }^{2}$ UCL Institute of Ophthalmology, London, UK. ${ }^{3}$ Moorfields Eye Hospital, London, UK. ${ }^{4}$ Audiovestibular Medicine, National Hospital for Neurology and Neurosurgery, London, UK. ${ }^{5}$ UCL Ear Institute, London, UK. ${ }^{6} \mathrm{CHU}$ Montpellier, Laboratoire de Génétique Moléculaire, Montpellier F-34000, France. ${ }^{7}$ Inserm, U827, Montpellier F-34000, France. ${ }^{8}$ Great Ormond Street Hospital, London, UK.

Received: 16 April 2013 Accepted: 4 August 2013 Published: 8 August 2013

\section{References}

1. Millán JM, Aller E, Jaijo T, Blanco-Kelly F, Gimenez-Pardo A, Ayuso C: An update on the genetics of usher syndrome. J Ophthalmol 2011, 2011:417217.

2. Saihan Z, Webster AR, Luxon L, Bitner-Glindzicz M: Update on Usher syndrome. Curr Opin Neurol 2009, 22:19-27.

3. Riazuddin S, Belyantseva IA, Giese APJ, Lee K, Indzhykulian AA, Nandamuri SP, Yousaf R, Sinha GP, Lee S, Terrell D, Hegde RS, Ali RA, Anwar S, AndradeElizondo PB, Sirmaci A, Parise LV, Basit S, Wali A, Ayub M, Ansar M, Ahmad W, Khan SN, Akram J, Tekin M, Riazuddin S, Cook T, Buschbeck EK, Frolenkov 
GI, Leal SM, Friedman TB, Ahmed ZM: Alterations of the CIB2 calcium- and integrin-binding protein cause Usher syndrome type $1 \mathrm{~J}$ and nonsyndromic deafness DFNB48. Nat Genet 2012, 44:1265-1271.

4. Ebermann I, Phillips JB, Liebau MC, Koenekoop RK, Schermer B, Lopez I, Schäfer E, Roux A-F, Dafinger C, Bernd A, Zrenner E, Claustres M, Blanco B, Nürnberg G, Nürnberg P, Ruland R, Westerfield M, Benzing T, Bolz HJ: PDZD7 is a modifier of retinal disease and a contributor to digenic Usher syndrome. J Clin Invest 2010, 120:1812-1823.

5. Kimberling WJ, Hildebrand MS, Shearer AE, Jensen ML, Halder JA, Trzupek K, Cohn ES, Weleber RG, Stone EM, Smith RJH: Frequency of Usher syndrome in two pediatric populations: implications for genetic screening of deaf and hard of hearing children. Genet Med 2010, 12:512-516.

6. Baux D, Larrieu L, Blanchet C, Hamel C, Ben Salah S, Vielle A, GilbertDussardier B, Holder M, Calvas P, Philip N, Edery P, Bonneau D, Claustres M, Malcolm S, Roux A-F: Molecular and in silico analyses of the full-length isoform of usher in identify new pathogenic alleles in Usher type II patients. Hum Mutat 2007, 28:781-789.

7. Le Quesne SP, Saihan Z, Rangesh N, Steele-Stallard HB, Ambrose J, Coffey A, Emmerson J, Haralambous E, Hughes Y, Steel KP, Luxon LM, Webster AR, Bitner-Glindzicz M: Comprehensive sequence analysis of nine Usher syndrome genes in the UK National Collaborative Usher Study. J Med Genet 2012, 49:27-36.

8. Garcia-Garcia G, Aparisi MJ, Jaijo T, Rodrigo R, Leon AM, Avila-Fernandez A, Blanco-Kelly F, Bernal S, Navarro R, Diaz-Llopis M, Baiget M, Ayuso C, Millan $J \mathrm{M}$, Aller E: Mutational screening of the USH2A gene in Spanish USH patients reveals 23 novel pathogenic mutations. Orphanet J Rare Dis 2011, 6:65.

9. USH-Bases: LSDBs for usher syndrome genes. https://grenada.lumc.nI/LOVD2/ Usher_montpellier/USHbases.html.

10. Roux A-F, Faugère $V$, Vaché $C$, Baux $D$, Besnard $T$, Léonard $S$, Blanchet $C$, Hamel C, Mondain M, Gilbert-Dussardier B, Edery P, Lacombe D, Bonneau D, Holder-Espinasse M, Ambrosetti U, Journel H, David A, Lina-Granade G, Malcolm S, Claustres M: Four-year follow-up of diagnostic service in USH1 patients. Invest Ophthalmol Vis Sci 2011, 52:4063-4071.

11. Vaché C, Besnard T, le Berre P, García-García G, Baux D, Larrieu L, Abadie C, Blanchet C, Bolz HJ, Millan J, Hamel C, Malcolm S, Claustres M, Roux A-F: Usher syndrome type 2 caused by activation of an USH2A pseudoexon: implications for diagnosis and therapy. Hum Mutat 2012, 33:104-108.

12. Nakanishi H, Ohtsubo M, Iwasaki S, Hotta Y, Mizuta K, Mineta H, Minoshima $\mathrm{S}$ : Hair roots as an mRNA source for mutation analysis of Usher syndrome-causing genes. J Hum Genet 2010, 55:701-703.

13. Vaché $C$, Besnard $T$, Blanchet $C$, Baux $D$, Larrieu L, Faugère V, Mondain $M$, Hamel C, Malcolm S, Claustres M, Roux A-F: Nasal epithelial cells are a reliable source to study splicing variants in Usher syndrome. Hum Mutat 2010, 31:734-741.

14. Aller E, Jaijo T, García-García G, Aparisi MJ, Blesa D, Díaz-Llopis M, Ayuso C, Millán JM: Identification of large rearrangements of the PCDH15 gene by combined MLPA and a CGH: large duplications are responsible for Usher syndrome. Invest Ophthalmol Vis Sci 2010, 51:5480-5485.

15. Besnard T, Vaché C, Baux D, Larrieu L, Abadie C, Blanchet C, Odent S, Blanchet P, Calvas P, Hamel C, Dollfus H, Lina-Granade G, Lespinasse J, David A, Isidor B, Morin G, Malcolm S, Tuffery-Giraud S, Claustres M, Roux AF: Non-USH2A mutations in USH2 patients. Hum Mutat 2012, 33:504-510.

16. MRC-Holland - MLPA procedure - introduction. http://www.mlpa.com/WebForms/ WebFormMain.aspx?Tag=wl2zCjilrCGANQgZPuTixsEyIW1MscfzuKj2NDFYclg|.

17. Softgenetics - software power tools for genetic analysis. http://www. softgenetics.com/GeneMarker.html.

18. Bonnet C, Grati M, Marlin S, Levilliers J, Hardelin J-P, Parodi M, Niasme-Grare $M$, Zelenika D, Délépine $M$, Feldmann $D$, Jonard L, El-Amraoui A, Weil D, Delobel B, Vincent C, Dollfus H, Eliot M-M, David A, Calais C, Vigneron J, Montaut-Verient B, Bonneau D, Dubin J, Thauvin C, Duvillard A, Francannet C, Mom T, Lacombe D, Duriez F, Drouin-Garraud V, Thuillier-Obstoy M-F, Sigaudy S, Frances A-M, Collignon P, Challe G, Couderc R, Lathrop M, Sahel J-A, Weissenbach J, Petit C, Denoyelle F: Complete exon sequencing of all known Usher syndrome genes greatly improves molecular diagnosis. Orphanet J Rare Dis 2011, 6:21.

19. Abu-Safieh L, Al-Anazi S, Al-Abdi L, Hashem M, Alkuraya H, Alamr M, Sirelkhatim MO, Al-Hassnan Z, Alkuraya B, Mohamed JY, Al-Salem A, Alrashed M, Fageih E, Softah A, Al-Hashem A, Wali S, Rahbeeni Z, Alsayed M, Khan AO, Al-Gazali L, Taschner PEM, Al-Hazzaa S, Alkuraya FS: In search of triallelism in Bardet-Biedl syndrome. Eur J Hum Genet 2012, 20:420-427.
20. Jaijo T, Aller E, García-García G, Aparisi MJ, Bernal S, Avila-Fernández A, Barragán I, Baiget M, Ayuso C, Antiñolo G, Díaz-Llopis M, Külm M, Beneyto M, Nájera C, Millán JM: Microarray-based mutation analysis of 183 Spanish families with Usher syndrome. Invest Ophthalmol Vis Sci 2010, 51:1311-1317.

21. Xu W, Dai H, Lu T, Zhang X, Dong B, Li Y: Seven novel mutations in the long isoform of the USH2A gene in Chinese families with nonsyndromic retinitis pigmentosa and Usher syndrome Type II. Mol Vis 2011, 17:1537-1552.

22. Saihan Z, Stabej PLQ, Robson AG, Rangesh N, Holder GE, Moore AT, Steel KP, Luxon LM, Bitner-Glindzicz M, Webster AR: Mutations in the USH1C gene associated with sector retinitis pigmentosa and hearing loss. Retina 2011, 31:1708-1716.

23. Hirami Y, Osakada F, Takahashi K, Okita K, Yamanaka S, Ikeda H, Yoshimura $\mathrm{N}$, Takahashi M: Generation of retinal cells from mouse and human induced pluripotent stem cells. Neurosci Lett 2009, 458:126-131.

24. Jin Z-B, Okamoto S, Osakada F, Homma K, Assawachananont J, Hirami Y, Iwata T, Takahashi M: Modeling retinal degeneration using patientspecific induced pluripotent stem cells. PLOS ONE 2011, 6:e17084.

25. Zahabi A, Shahbazi E, Ahmadieh H, Hassani S-N, Totonchi M, Taei A, Masoudi N, Ebrahimi M, Aghdami N, Seifinejad A, Mehrnejad F, Daftarian N, Salekdeh GH, Baharvand H: A new efficient protocol for directed differentiation of retinal pigmented epithelial cells from normal and retinal disease induced pluripotent stem cells. Stem Cells Dev 2012, 21:2262-2272

26. Zou J, Luo L, Shen Z, Chiodo VA, Ambati BK, Hauswirth WW, Yang J: Whirlin replacement restores the formation of the USH2 protein complex in whirlin knockout photoreceptors. Invest Ophthalmol Vis Sci 2011, 52:2343-2351.

27. Goldmann T, Overlack N, Möller F, Belakhov V, van Wyk M, Baasov T, Wolfrum U, Nagel-Wolfrum K: A comparative evaluation of NB30, NB54 and PTC124 in translational read-through efficacy for treatment of an USH $1 \mathrm{C}$ nonsense mutation. EMBO Mol Med 2012

28. Overlack N, Goldmann T, Wolfrum U, Nagel-Wolfrum K: Gene repair of an Usher syndrome causing mutation by zinc-finger nuclease mediated homologous recombination. Invest Ophthalmol Vis Sci 2012, 53:4140-4146.

29. Williams DS: Usher syndrome: animal models, retinal function of Usher proteins, and prospects for gene therapy. Vision Res 2008, 48:433-441.

30. Sahly I, Dufour E, Schietroma C, Michel V, Bahloul A, Perfettini I, Pepermans E, Estivalet A, Carette D, Aghaie A, Ebermann I, Lelli A, Iribarne M, Hardelin JP, Weil D, Sahel J-A, El-Amraoui A, Petit C: Localization of Usher 1 proteins to the photoreceptor calyceal processes, which are absent from mice. J Cell Biol 2012, 199:381-399.

doi:10.1186/1750-1172-8-122

Cite this article as: Steele-Stallard et al:: Screening for duplications, deletions and a common intronic mutation detects $35 \%$ of second mutations in patients with USH2A monoallelic mutations on Sanger sequencing. Orphanet Journal of Rare Diseases 2013 8:122.

\section{Submit your next manuscript to BioMed Central and take full advantage of:}

- Convenient online submission

- Thorough peer review

- No space constraints or color figure charges

- Immediate publication on acceptance

- Inclusion in PubMed, CAS, Scopus and Google Scholar

- Research which is freely available for redistribution 